Check for updates

Cite this: RSC Adv., 2018, 8, 31777

Received 11th July 2018

Accepted 27th August 2018

DOI: 10.1039/c8ra05921g

rsc.li/rsc-advances

\title{
Synthesis of non-ionic bolaamphiphiles and study of their self-assembly and transport behaviour for drug delivery applications $\dagger$
}

\author{
Rashmi, ${ }^{a}$ Abhishek K. Singh, ${ }^{a}$ Katharina Achazi, ${ }^{b}$ Boris Schade, (D) ${ }^{c}$ \\ Christoph Böttcher, ${ }^{c}$ Rainer Haag ${ }^{b}$ and Sunil K. Sharma (iD *a
}

\begin{abstract}
A series of four bolaamphiphiles with different hydrophilic units has been synthesised. All the amphiphiles were well characterised from their physiochemical data. The aggregation tendency of newly synthesised amphiphiles was studied using fluorescence spectroscopy, dynamic light scattering (DLS), and cryogenic electron microscopy (cryo-TEM). Furthermore, their application as nanocarriers for hydrophobic guests was demonstrated by using two established standards, i.e. the dye Nile red and the drug nimodipine. A cytotoxicity and cellular uptake study has been carried out using A549 cells. Due to the presence of an ester linkage in PEG based bolaamphiphiles, a drug release study was performed in the presence of an immobilized enzyme Novozym-435 (a lipase).
\end{abstract}

\section{Introduction}

Inspired by nature, the self-assembly of amphiphilic molecules mimics various biological systems depending on their structure, concentration, solution environment, and temperature. The spontaneous organization of sophisticated molecules like lipids, proteins, saccharides and nucleic acids provides vital information to understand the factors responsible for their self-assembly. Various non-covalent interactions like hydrogen bonding, hydrophobic interactions, $\pi-\pi$ stacking, etc. provide the momentum for self-aggregation. In recent years, this concept has largely been used for various applications in biomedical and material sciences. ${ }^{1-8}$ As many drugs suffer from limited aqueous-solubility and shorter half-life in the bloodstream, resulting in their limited bioavailability, the supramolecular nano-carriers have shown promising behaviour in the target specific drug delivery. For the enhanced therapeutic action of such drugs, these are encapsulated in the hydrophobic core of the amphiphiles and the hydrophilic groups surfaced the assembly to keep the guest intact from aqueous medium and solubilize the whole molecule. ${ }^{9-18}$ For the past decades the amphiphilic architectures have earned considerable attention to such delivery systems. To explore the usefulness of such systems, new amphiphilic architectures have been designed

${ }^{a}$ Department of Chemistry, University of Delhi, Delhi 110 007, India. E-mail: sksharma@chemistry.du.ac.in; Tel: +91-11-27666646

${ }^{b}$ Institut für Chemie und Biochemie, Freie Universität Berlin, Takustraße 3, 14195 Berlin, Germany. E-mail: haag@zedat.fu-berlin.de; Fax: +49-30-838-452633; Tel: +49-30-838-52633

${ }^{c}$ Forschungszentrum für Elektronenmikroskopie, Institut für Chemie und Biochemie, Freie Universität Berlin, Fabeckstraße 36a, 14195 Berlin, Germany

$\dagger$ Electronic supplementary information (ESI) available. See DOI: 10.1039/c8ra05921g that can be used as nano-carriers for encapsulation and release of drug/dyes in a systematic manner. Among various amphiphilic architectures studied, bolaamphiphiles with two hydrophilic end groups separated by a hydrophobic spacer, exhibit unique hierarchically self-assembled structures from micelles to large cylindrical, vesical and monolayer formation at air-water interface. Furthermore, compared to the conventional surfactants, bolaamphiphiles have higher aqueous solubility and reduced critical aggregation concentration. ${ }^{\mathbf{1 9}, 20}$ Although a number of ionic and non-ionic bolaamphiphiles constituted from carbohydrates and amino acids have been reported for various pharmaceutical applications, ${ }^{21-27}$ however the bolaamphiphiles based on polyethylene glycol (PEG) and dendritic polyglycerol (dPG) are not very common besides being biocompatible, nontoxic, stable, and aqueous soluble. Furthermore, PEG and PG are known to evade recognition by the reticulo-endothelial system (RES), which in turn qualify them for use in drug delivery applications. ${ }^{28-35}$

Herein we report the synthesis of four different pegylated and dendronized symmetrical bolaamphiphiles by coupling together, a novel hydrophobic aryl-alkyne conjugate with different hydrophilic PEG/dPG azides by following the CuAAC methodologies (Fig. 1). The synthesized bolaamphiphiles were characterized using NMR, IR, HRMS and GPC. The aggregation behaviour of amphiphiles was studied using DLS and cryo-TEM. Their drug encapsulation potential and release profile was studied using UV and fluorescence spectroscopy.

\section{Results and discussion}

\section{Synthesis and characterization}

The chemical and physical properties of amphiphilic systems vary with respect to their hydrophobic and hydrophilic content. 


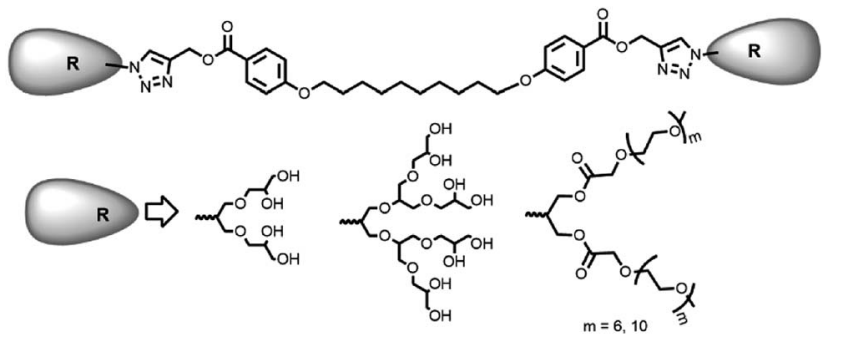

Fig. 1 Polyethyleneglycol (PEG) and dendritic polyglycerol (dPGs) based bolaamphiphiles.

To systematically explore the influence of hydrophilic groups on aggregation behaviour, i.e. size, morphology, encapsulation and release profile of guests, etc., bolaamphiphiles with different polar head groups were synthesised by coupling together hydrophobic and hydrophilic moiety using CuAAC methodologies. Four symmetrical bolaamphiphiles have been synthesised by utilizing PEG $\left(M_{\mathrm{n}}: 350 / 550\right)$ and dendritic polyglycerol [G1.0/ G2.0] as hydrophilic moieties. The synthesis of hydrophilic groups 6/7 involves acylation of azidodiol 5 with mono methoxy PEG acid [( $\left.M_{\mathrm{n}}: 350 / 550\right) 3 / 4$ ], mPEG acids (3/4) and azidodiol were in turn prepared by following literature procedure (Scheme 1). ${ }^{36}$ G1.0 and G2.0 dendritic polyglycerol azides (dPGs azides) were also synthesised by following literature procedure. ${ }^{37}$ For the synthesis of the hydrophobic core, $p$-hydroxybenzoic acid was first converted quantitatively to its propargyl ester 8 by using propargyl bromide in the presence of a mild base. Compound 8 was then reacted with 1,10-dibromodecane on its both ends to yield the desired diacetylinic product 9. The hydrophobic alkyne and PEG/dPG azides were then subjected to click reaction to obtain the target bolaamphiphiles (10-13) (Scheme 2). All the bolaamphiphiles synthesized were characterized on the basis of their physical and spectral data i.e. ${ }^{1} \mathrm{H}$ and, ${ }^{13} \mathrm{C}-\mathrm{NMR}$ spectroscopy, HRMS and gel permeation chromatography (GPC).

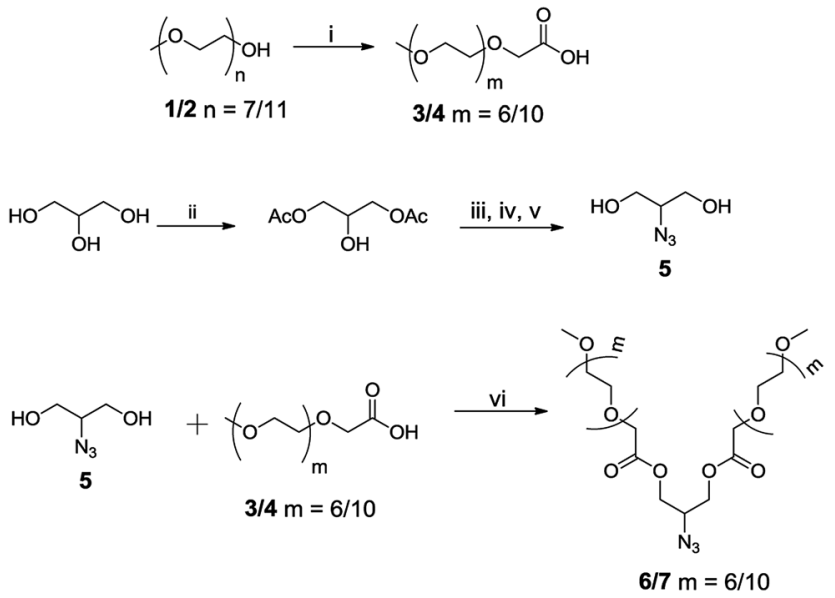

Scheme 1 Synthesis of hydrophilic segments; (i) $\mathrm{KMnO}_{4}$, water, $80^{\circ} \mathrm{C}$, 24 h; (ii) Novozym-435, vinyl acetate, THF, $37^{\circ} \mathrm{C}$; (iii) mesylchloride, triethylamine, DCM, $0-25^{\circ} \mathrm{C}, 2 \mathrm{~h}$; (iv) $\mathrm{NaN}_{3}, \mathrm{DMF}$, reflux, $12 \mathrm{~h}$; (v) $\mathrm{KOH}$, $\mathrm{EtOH}, 12 \mathrm{~h}$; (vi) EDC.HCl, DMAP, DCM, $35^{\circ} \mathrm{C}, 12 \mathrm{~h}$.

\section{Critical aggregation concentration (CAC)}

The CAC of bolaamphiphiles (10-13) was determined by fluorescence measurement using a hydrophobic dye Nile red. The assembly was initially probed with Nile red by dissolving respective bolaamphiphiles in water. The plot of fluorescence intensity of encapsulated Nile red versus $\log$ [amphiphile conc.] gave the CAC value at the point of inflection, and it was observed to be in the range of $1.5 \times 10^{-4}$ to $9.2 \times 10^{-5} \mathrm{M}$ (Table 1). Employing the Griffin equation ${ }^{38}$ for calculating HLB (Hydrophilic-Lipophilic Balance) shows that in the pegylated bolaamphiphiles Bola-PEG 6 with low HLB exhibit higher CAC value as compared to the Bola- PEG $_{10}$ having an enhanced hydrophilic character. A similar behaviour was observed with dendronized bolaamphiphiles i.e. Bola-[G2.0] having a higher HLB exhibit lower CAC as compared to Bola-[G1.0] (Fig. S16, see the ESI $\dagger$ ).

\section{Dynamic light scattering (DLS) and cryo-TEM measurement}

The hydrodynamic size of the particles was determined by dynamic light scattering (DLS) technique at a concentration of $5.0 \mathrm{mg} \mathrm{mL} \mathrm{m}^{-1}$. The particle size of the nanoparticles in the aqueous solution obtained from DLS was observed to be in the range 4 to $7 \mathrm{~nm}$ (Fig. 2) for all the four bolaamphiphiles synthesized. The size distribution was monomodal in volume and number, but bimodal in intensity. In the case of bimodular system, the two peaks were assigned for molecular aggregates assemblies, whereas single peaks in volume and number corresponds to simple molecular assembly in aqueous medium, indicating that mainly small size particles are formed as compared to larger molecular aggregates. Nanoparticles of up to $200 \mathrm{~nm}$ size show prolonged delivery of therapeutic agents and exhibit higher drug uptake compared to microparticles. ${ }^{39}$ The nano sized aggregates formed by the bolaamphiphiles in this study were found to be of appropriate size for studying their biological applications. As the amphiphiles in aqueous medium agglomerate, so the actual size and morphology cannot be evaluated rather a hydrodynamic size is obtained from the DLS study. For the precise measurement of particle size and to visualize the actual morphology in aqueous medium cryo-TEM technique was utilized for one of the representative amphiphile Bola-[G1.0]. The cryo-TEM image for Bola-[G1.0] (Fig. 3) shows small elongated aggregates with an average diameter of $\sim 3 \mathrm{~nm}$, which is in good agreement with the DLS data by considering the fact that the hydrodynamic size is generally greater than the visible assembly structure. ${ }^{\mathbf{4 0}}$ Such smaller molecular aggregates are of optimum size to be considered for therapeutic applications.

\section{Cytotoxicity study}

Cytotoxicity of the bolaamphiphiles was assessed by an MTS assay using A549 lung cancer cells. All of the amphiphiles showed a similar trend i.e. low toxicity at the highest test concentration of $1 \mathrm{mg} \mathrm{mL}^{-1}$ (Fig. 4). However, at $0.1 \mathrm{mg} \mathrm{mL}^{-1}$, all the bolaamphiphiles were found to be non-cytotoxic and thus suitable for biological applications. 


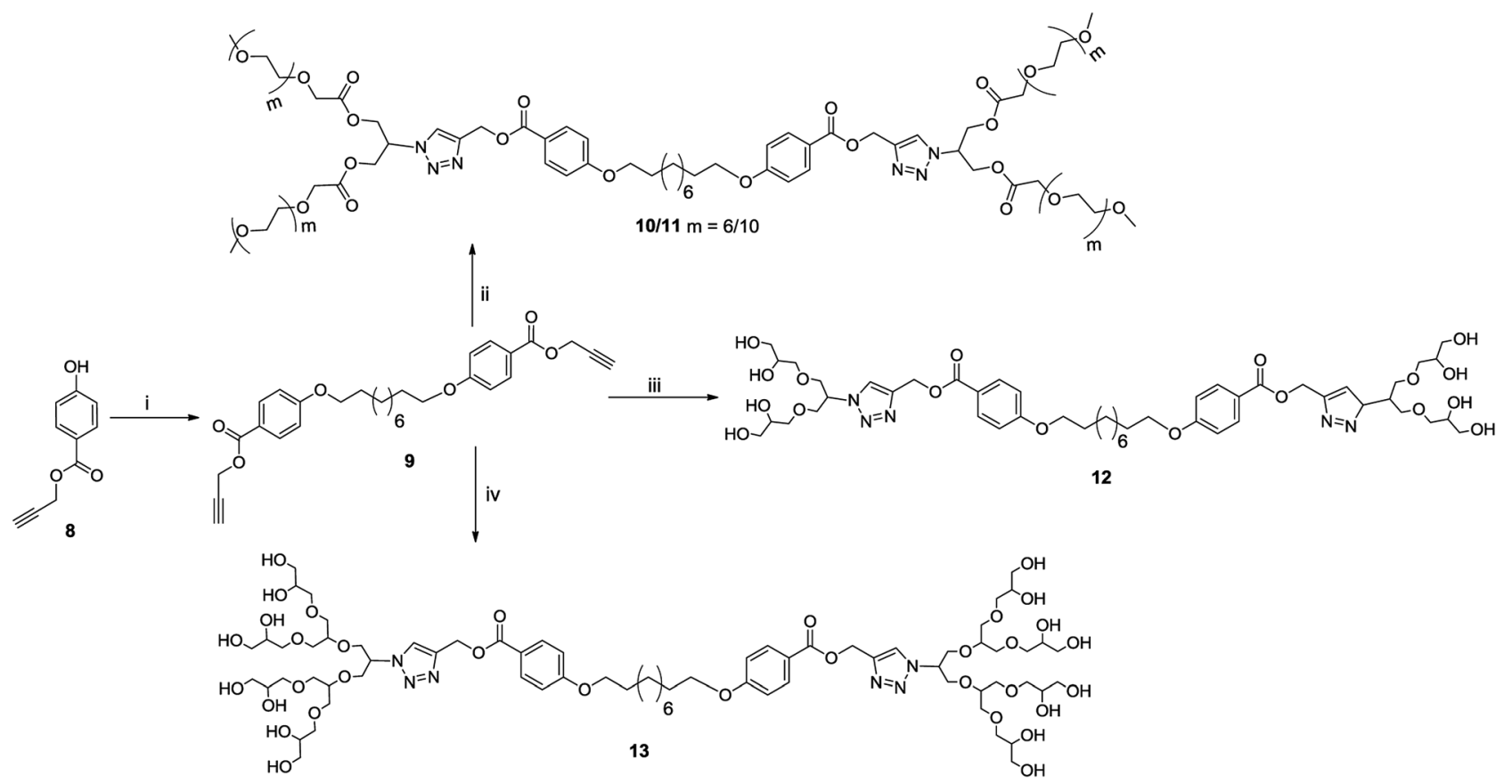

Scheme 2 Synthesis of target bolaamphiphiles; (i) 1,10-dibromodecane, $\mathrm{K}_{2} \mathrm{CO}_{3}, 35{ }^{\circ} \mathrm{C}, 15 \mathrm{~h}$; (ii) compound 6/7, copper sulphate, sodium ascorbate, THF : water (3 : 1), $50{ }^{\circ} \mathrm{C}, 24 \mathrm{~h}$; (iii) [G1.0] azide, copper sulphate, sodium ascorbate, THF : water, $50{ }^{\circ} \mathrm{C}, 24 \mathrm{~h}$; (iv) [G2.0] azide, copper sulphate, sodium ascorbate, THF : water, $50^{\circ} \mathrm{C}, 24 \mathrm{~h}$.

Table 1 Aggregation study of synthesized bolaamphiphiles

\begin{tabular}{lrrll}
\hline Bolaamphiphile & HLB & CAC (M) & DLS (nm) & Cryo-TEM (nm) \\
\hline Bola-PEG $_{6}$ & 14.44 & $1.75 \times 10^{-4}$ & 7 & - \\
Bola-PEG $_{10}$ & 15.85 & $1.50 \times 10^{-4}$ & 6 & - \\
Bola-[G1.0] & 8.74 & $2.14 \times 10^{-4}$ & 5 & $2-3^{a}$ \\
Bola-[G2.0] & 12.87 & $9.2 \times 10^{-5}$ & 4 & -
\end{tabular}

${ }^{a}$ Performed on one representative amphiphile.

\section{Encapsulation study}

Nile red encapsulation. The impetus of self-organisation of the non-ionic bolaamphiphiles in aqueous medium forming a hydrophobic inner core renders the possibility to encapsulate guest dye/drug in its hydrophobic core by non-covalent interactions like $\pi-\pi$ stacking, hydrogen bonding, hydrophobic effects, etc. In the light of these effects, the encapsulation potential of bolaamphiphiles was determined by employing the film method and taking Nile red as a model probe. Due to its limited aqueous solubility and high solvatochromic fluorescence with strong emission in the lipophilic environment, Nile red can be used to divulge useful information about the encapsulation efficiency and site of encapsulation. Based on the various literature reports and our own experience, $0.12 \mathrm{mg}$ of Nile red was encapsulated in $5 \mathrm{mg} \mathrm{mL}^{-1}$ of amphiphiles to avoid the possibility of aggregation of the dye and this results in maximum encapsulation of the guest molecule. ${ }^{\mathbf{4 1 , 4 2}}$ The quantification of Nile red encapsulation was carried by lyophilisation of the dye encapsulated samples followed by the dissolution of contents in methanol $(1 \mathrm{~mL})$ with subsequent measurement of their absorbance by UV-vis spectroscopy. The transport capacity of all the amphiphiles was calculated employing BeerLambert's law and using a molar extinction coefficient of $45000 \mathrm{M}^{-1} \mathrm{~cm}^{-1}$ at $552 \mathrm{~nm}$ for the dye. In accordance with the HLB values, the dendronized bolaamphiphiles constituted from [G1.0] dendron (Bola-[G1.0]) exhibit higher encapsulation of Nile red $5.68 \mathrm{mg} \mathrm{g}^{-1}$ as compared to its analogue Bola-[G2.0]. Similarly, among the pegylated bolaamphiphiles, the one with a higher lipophilic ratio, i.e. Bola- $\mathrm{PEG}_{6}$ encapsulated more amount of Nile red as compared to Bola-PEG 10 (Fig. 5).

Nimodipine encapsulation. Nimodipine, a potent calcium channel blocker, is a hydrophobic drug with a limited aqueous solubility of $3.86 \mu \mathrm{g} \mathrm{mL}^{-1}$, that renders low bioavailability of the drug. ${ }^{43}$ To address this issue, Nimodipine was encapsulated in the nanoaggregates fabricated from the bolaamphiphiles in aqueous medium by following the thin film method. The quantification of nimodipine per gram of bolaamphiphile was measured using UV spectrophotometer following BeerLambert's law using an extinction coefficient of nimodipine as $7200 \mathrm{M}^{-1} \mathrm{~cm}^{-1}$ at $365 \mathrm{~nm}$ in ethanol. Bola- $\mathrm{PEG}_{6}$ shows higher loading capacity than Bola- $\mathrm{PEG}_{10}$, whereas in the case of dendronized amphiphiles, Bola-[G2.0] shows higher loading capacity of nimodipine as compared to Bola-[G1.0] (Fig. 5).

\section{Cellular uptake study}

Confocal laser scanning microscopy was used to analyse the cellular uptake behaviour of Nile red loaded bolaamphiphiles Bola-PEG 6 and Bola-[G1.0] to simulate drug transport by the amphiphiles. A test concentration of $0.1 \mathrm{mg} \mathrm{mL}^{-1}$ of the bolaamphiphiles was chosen considering the toxicity study 

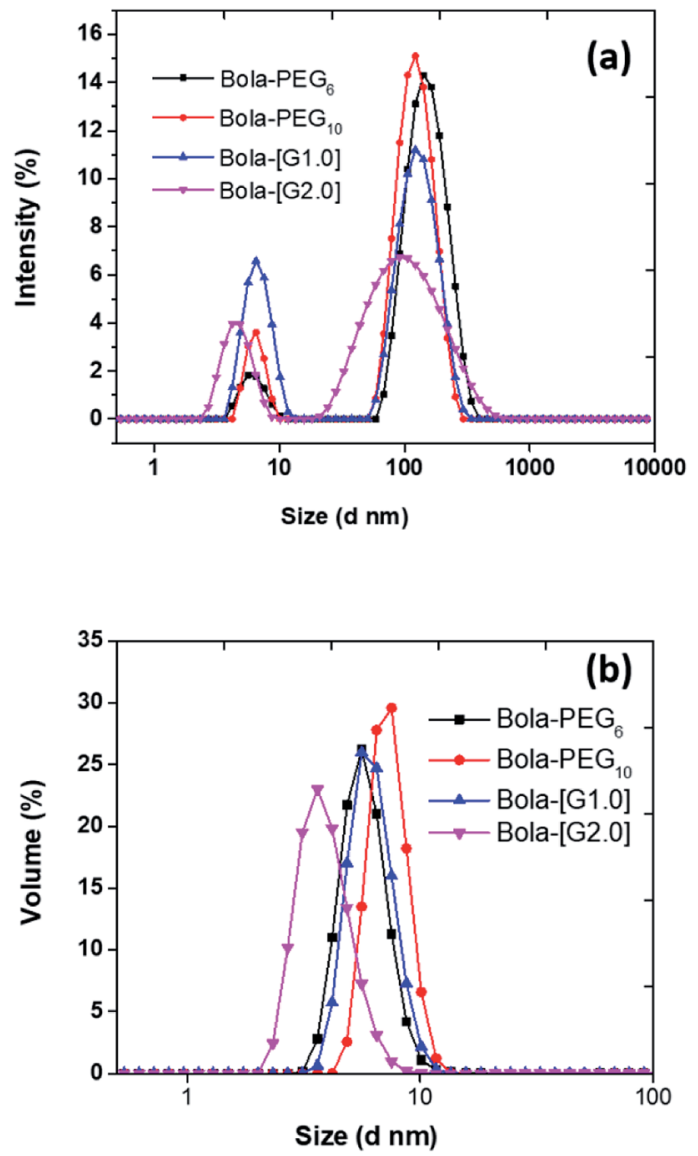

Fig. 2 Plot of (a) intensity versus size; (b) volume versus size for calculating hydrodynamic size $(\mathrm{nm})$ of bolaamphiphiles obtained from dynamic light scattering (DLS).

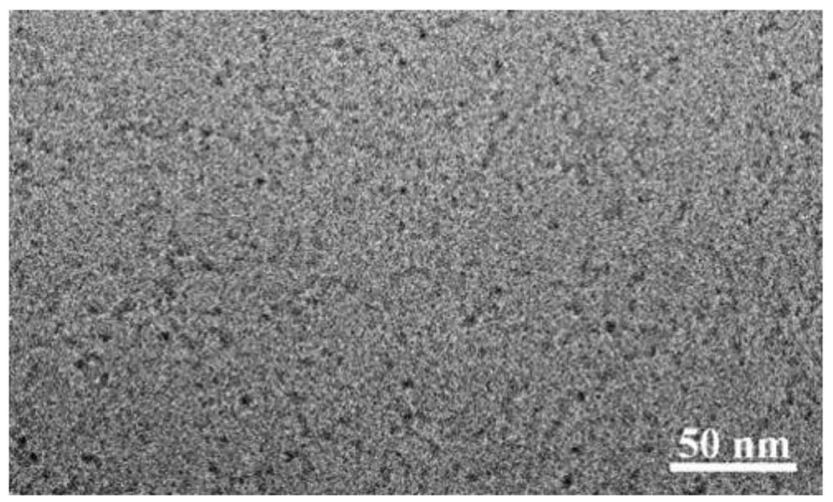

Fig. 3 Cryo-TEM micrograph of Bola-[G1.0].

results. Both the amphiphiles exhibit a similar uptake pattern of Nile red inside the cell. Cellular uptake in the cell was seen even after the first hour of incubation (Fig. 6). After $24 \mathrm{~h}$, the bolaamphiphiles were observed to be accumulated in the cells' cytosol. However, no Nile red was visible in the nucleus. The results indicate that the amphiphiles are well suited to carry drugs in the cytosol of cells.

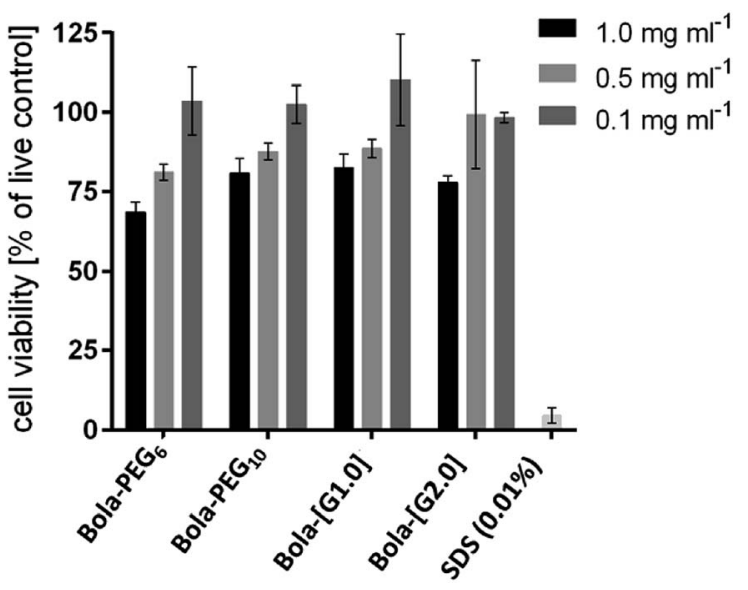

Fig. 4 Cytotoxicity profile of the bolaamphiphiles after 24 h. Cytotoxicity of the amphiphiles was determined by an MTS assay using A549 cells with $24 \mathrm{~h}$ post-treatment. Each bar represents the mean value of triplicate study with SD.

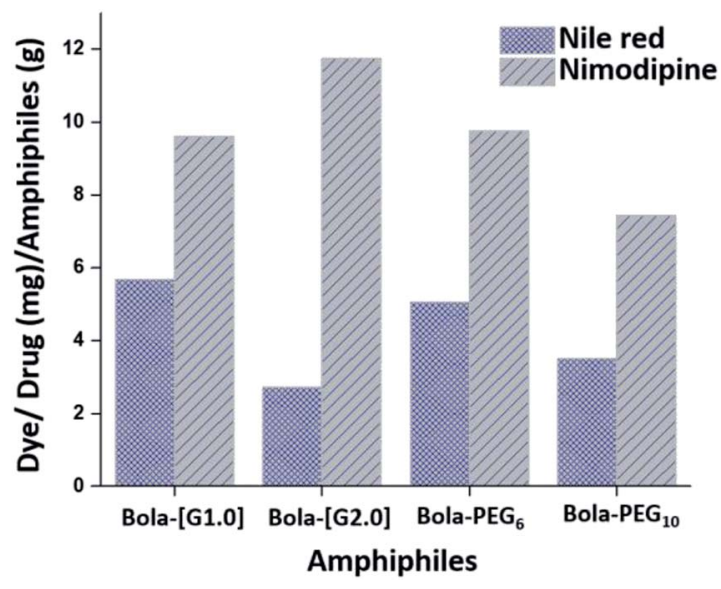

Fig. 5 Transport capacity of bolaamphiphiles.

\section{Enzyme-triggered release of encapsulated guest}

Besides encapsulation, the release of encapsulated therapeutic entity is also significantly important. We studied the enzyme triggered release of the encapsulated guest from the inner hydrophobic core of amphiphilic aggregates. Since the amphiphiles Bola- $\mathrm{PEG}_{6}$ and Bola- $\mathrm{PEG}_{10}$ constitute from ester functionality, we studied the enzyme mediated ester cleavage. Such an act can be triggered in biological systems as a number of hydrolytic enzymes are available over there.

We performed the enzyme mediated release study using the same hydrolytic enzyme that was used for the synthesis of bolaamphiphiles i.e. immobilized Candida antarctica lipase (Novozym-435). The release study was performed in dark and at $37{ }^{\circ} \mathrm{C}$. It was observed that within $5 \mathrm{~h}$ of enzyme exposure a complete release of encapsulated dye takes place, however the control experiment performed without the enzyme does not exhibit any noticeable release of encapsulated dye (Fig. 7). 


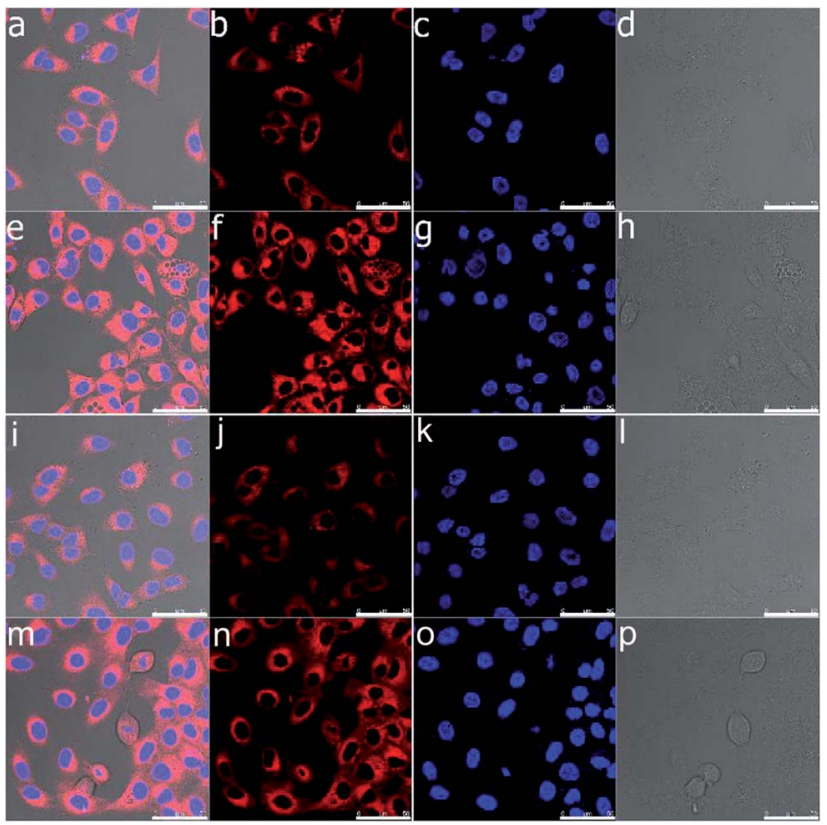

Fig. 6 Confocal laser scanning microscopy images of A549 cells after 1 hour $(a-d$ and $i-l)$ and $24 \mathrm{~h}(e-h$ and $m-p)$ incubation with Nile red encapsulated by bolaamphiphiles Bola-PEG $6(a-h)$ and Bola-[G1.0] (ip). In the images, Nile red is shown in red color and the nucleus stained with Hoechst 33342 in blue. The bright field channel is shown in grey scale. The scale bar equals $50 \mu \mathrm{m}$

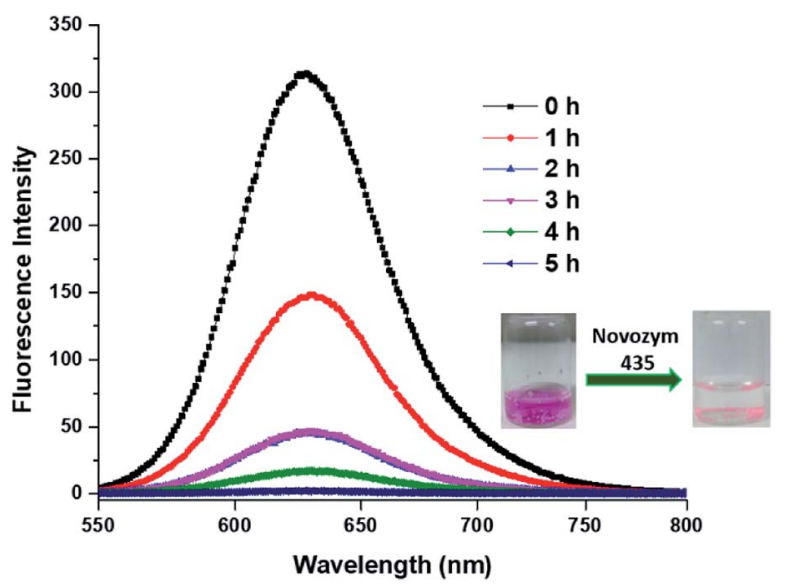

Fig. 7 Enzymatic mediated release of encapsulated dye Nile red from Bola-PEG6.

\section{Conclusions}

A set of four linear and dendronized bolaamphiphiles have been synthesised in a chemo-enzymatic manner by employing the immobilized enzyme Novozym 435 and following a CuAAC methodologies. Furthermore, biocompatible materials like polyethylene glycol (PEG) and polyglycerol dendrons (dPGs) were used as building blocks. The bolaamphiphiles synthesised were well characterized from their spectroscopic and physical data, e.g. ${ }^{1} \mathrm{H}-\mathrm{NMR},{ }^{13} \mathrm{C}-\mathrm{NMR}$, HRMS, and GPC techniques. Fabrication of nano-carrier has been achieved by allowing the amphiphile aggregation in aqueous medium. The size of the aggregates was studied using DLS and cryo-TEM. Nile red as a model dye and nimodipine as a model drug were used for evaluating the encapsulation potential of amphiphiles.

Bola-PEG 6 and Bola-[G1.0] exhibit a higher encapsulation tendency than their larger pegylated and dendronized analogues i.e. Bola- $\mathrm{PEG}_{10}$ and Bola-[G2.0] respectively. The enzyme mediated release of the encapsulated dye was also studied using Bola- $\mathrm{PEG}_{6}$, which shows an efficient dye release from the hydrophobic core of the amphiphile. Cell viability study shows that all bolaamphiphiles are well tolerated upto a concentration of $0.5 \mathrm{mg} \mathrm{mL}{ }^{-1}$. In conclusion the good biocompatibility, the higher drug loading capacity and fast enzymatic release shows that the synthesised bolaamphiphiles can be used as a starting point to design and develop efficient nanocarriers for drugs and dyes.

\section{Conflicts of interest}

There is no conflicts of interest to be declare.

\section{Acknowledgements}

We gratefully acknowledge the DST, Government of India and DFG, Germany and the core facility BioSupraMol, FU-Berlin for supporting a collaborative research project financial assistance and UGC-CSIR, New Delhi, for providing fellowship to Rashmi.

\section{References}

1 M. Ramanathan, L. K. Shrestha, T. Mori, Q. Ji, J. P. Hill and K. Ariga, Phys. Chem. Chem. Phys., 2013, 15, 10580-10611.

2 A. Sorrenti, O. Illa and R. M. Ortun, Chem. Soc. Rev., 2013, 42, 8200-8219.

3 P. A. Albouy, S. Deville, A. Fulkar, K. Hakouk, M. ImperorClerc, M. Klotz, Q. Liu, M. Marcellinib and J. Perezd, Soft Matter, 2017, 13, 1759-1763.

4 P. Calandra, D. Caschera, V. T. Liveri and D. Lombardoc, Colloids Surf., A, 2015, 484, 164-183.

5 M. Lee, B. K. Cho and W. C. Zin, Chem. Rev., 2001, 101, 38693892.

6 J. A. A. W. Elemans, A. E. Rowan and R. J. M. Nolte, J. Mater. Chem., 2003, 13, 2661-2670.

7 C. Wang, Z. Wang and X. Zang, Acc. Chem. Res., 2012, 45, 608-618.

8 Y. Tu, F. Peng, A. Adawy, Y. Men, L. K. E. A. Abdelmohsen and D. A. Wilson, Chem. Rev., 2016, 116, 2023-2078.

9 R. Lin and H. Cui, Curr. Opin. Chem. Eng., 2015, 7, 75-83.

10 R. Haag, Angew. Chem., Int. Ed., 2004, 43, 278-282.

11 H. Chen, C. Khemtong, X. Yang, X. Cjang and J. Gao, Drug Discovery Today, 2011, 16, 354-360.

12 P. Khadka, J. Ro, H. Kim, I. Kim, J. T. Kim, H. Kim, J. M. Cho, G. Yun and J. Lee, Asian J. Pharm. Sci., 2014, 9, 304-316.

13 N. A. Kotov, Nanostruct. Mater., 1999, 12, 789-796.

14 S. Prasad, K. Achazi, C. Bottcher, R. Haag and S. K. Sharma, $R S C$ Adv. , 2017, 7, 22121-22132. 
15 X. Hu, R. Liu, D. Zhang, J. Zhang, Z. Li and Y. Luan, ACS Biomater. Sci. Eng., 2018, 4, 973-980.

16 W. He, X. Hu, W. Jiang, R. Liu, D. Zhang, J. Zhang, Z. Li and Y. Luan, Adv. Healthcare Mater., 2017, 6, 1700829-1700842.

17 J. Zhang, D. Zhang, X. Hu, R. Liu, Z. Lib and Y. Luan, RSC Adv., 2018, 8, 13103-13111.

18 P. Zhang, H. Zhang, W. He, D. Zhao, A. Song and Y. Luan, Biomacromolecules, 2016, 17, 1621-1632.

19 M. Fariya, A. Jain, V. Dhawan, S. Shah and M. S. Nagarsenker, Adv. Pharm. Bull., 2014, 4, 483-491.

20 N. Nuraje, H. Bai and K. Su, Prog. Polym. Sci., 2013, 38, 302343.

21 S. Drescher, A. Meister, V. M. Garamus, G. Hause, C. J. Garvey, B. Dobner and A. Blume, Eur. J. Lipid Sci. Technol., 2014, 116, 1205-1216.

22 M. Popov, S. Grinberg, C. Linder, T. Waner, B. Levi-Hevroni, R. J. Deckelbaum and E. Heldman, J. Controlled Release, 2012, 160, 306-314.

23 Y. Jin, N. Qia, L. Tong and D. Chen, Int. J. Pharm., 2010, 386, 268-274.

24 C. Lee and S. Lee, RSC Adv., 2017, 7, 38989-38997.

25 Z. Khan, S. A. Al-Thabaiti and M. A. Malik, $R S C A d v ., 2016,6$, 45993-46001.

26 A. M. Reddy, A. Sharma, Q. Maqbool and A. Srivastava, RSC Adv., 2013, 3, 18900-18907.

27 S. Schmid, D. Y. W. Ng, E. Mena-Osteritz, Y. Wu, T. Weil and P. Bäuerle, Chem. Commun., 2016, 52, 3235-3238.

28 Y. Zhang, E. Mintzer and K. E. Uhrich, J. Colloid Interface Sci., 2016, 482, 19-26.

29 K. J. Harrington, G. Rowlinson-Busza, K. N. Syrigos, P. S. Uster, R. G. Vile and J. S. W. Stewar, Clin. Cancer Res., 2000, 6, 2528-2537.
30 T. M. Allen, C. Hansen, F. Martin, C. Redemann and A. YauYoung, Biochim. Biophys. Acta, 1991, 1066, 29-36.

31 B. Obermeie, F. Wurm, C. Mangold and H. Frey, Angew. Chem., Int. Ed., 2011, 50, 7988-7997.

32 S. Gupta, R. Tyagi, V. S. Parmar, S. K. Sharma and R. Haag, Polymer, 2012, 53, 3053-3078.

33 B. N. S. Thota, L. H. Urner and R. Haag, Chem. Rev., 2016, 116, 2079-2102.

34 A. K. Singh, B. N. S. Thota, B. Schade, K. Achazi, A. Khan, C. Böttcher, S. K. Sharma and R. Haag, Chem.-Asian J., 2017, 12, 1796-1806.

35 L. H. Urner, B. N. S. Thota, O. Nachtigall, S. Warnke, G. Helden, R. Haag and K. Pagel, Chem. Commun., 2015, 51, 8801-8804.

36 S. Gupta, B. Schade, S. Kumar, C. Böttcher, S. K. Sharma and R. Haag, Small, 2013, 9, 894-904.

37 M. Wyszogrodzka and R. Haag, Chem.-Eur. J., 2008, 14, 9202-9214.

38 W. C. Griffin, J. Soc. Cosmet. Chem., 1949, 1, 311-326.

39 R. Singh and J. W. Lillard Jr, Exp. Mol. Pathol., 2009, 86, 215223.

40 B. N. S. Thota, H. Berlepsch, C. Böttcher and R. Haag, Chem. Commun., 2015, 51, 8648-8651.

41 M. Kumari, S. Gupta, K. Achazi, C. Böttcher, J. Khandare, S. K. Sharma and R. Haag, Macromol. Rapid Commun., 2015, 36, 254-261.

42 M. Kumari, M. Billamboz, E. Leonard, C. Len, C. Böttcher, A. K. Prasad, R. Haag and S. K. Sharma, RSC Adv., 2015, 5, 48301-48310.

43 G. M. Soliman, R. Sharma, A. O. Choi, S. K. Varshney, F. M. Winnik, A. K. Kakkar and D. Maysinger, Biomaterials, 2010, 31, 8382-8392. 